\title{
Steady three dimensional gliding motion of an underwater glider
}

\author{
Shaowei Zhang, Jiancheng Yu, Aiqun Zhang, and Fumin Zhang
}

\begin{abstract}
Underwater Gliders have found broad applications in ocean sampling. In this paper, the nonlinear dynamic model of the glider developed by the Shenyang Institute of Automation, Chinese Academy of Sciences, is established. Based on this model, we solve for the parameters that characterize steady state spiraling motions of the glider. A set of nonlinear equations are simplified so that a recursive algorithm can be used to find the solutions.
\end{abstract}

\section{INTRODUCTION}

Gliders are autonomous controllable mobile platforms that use wing force and changing buoyancy as propulsion. Commercially available gliders include the Slocum [1], the Spray [2] and the Seaglider [3], which are capable of long range missions with low power consumption. Underwater gliders are employed in deep sea exploration [4] and ocean observation [5]-[7]. The dynamics of gliders are modeled in [8]-[11], and the straight line gliding motion in the vertical plane are stabilized by feedback control. The complex dynamic model and the coupled hydrodynamic effects make it difficult to find accurate solutions for the glider model that establishes relationships between the gliding state and the control input. The problem remains unsolved for three dimensional gliding motion such as the spiraling motion. In [9], a first attempt is made to find numerical solutions for the steady state equations that characterize the spiraling motion. In [12] and [13], an approximate analytical solution for steady spiraling motion is derived by applying perturbation theory.

The dynamic performance of an underwater glider is strongly affected by hydrodynamics. In [14], a thorough analysis about the hydrodynamic coefficients of a glider is performed. In [15], the drag and lift forces caused by wings of a fin-actuated underwater vehicle are studied. In [16], the hydrodynamic coefficients of autonomous underwater vehicles are verified using the computational fluid dynamics method and experiments. The effect of independently controllable wings on a glider is studied to enhance the glider maneuverability in [17].

The underwater glider developed by the Shenyang Institute of Automation (SIA) in [18] is propelled by a pump and steered by a movable-rotatable battery. The heave is controlled by the pump that changes the volume of a bladder.

This work is supported by the National High Technology Research and Development Program of China \#2006AA092157, State Key Laboratory of Robotics \#PLZ200810, and the Knowledge Innovation Program of the Chinese Academy of Sciences,Grant No.KZCX2-YW-JS205.

Shaowei Zhang, Jiancheng Yu, and Aiqun Zhang are with Shenyang Institute of Automation, Chinese Academy of Sciences. Shenyang 110016, Liaoning Province, China, $\{$ zhshw, yjc, zaq\}@sia.cn

Fumin Zhang is with Electrical and Computer Engineering, Georgia Institute of Technology, Atlanta, GA 30332, fumin@gatech. edu
The pitch and roll are controlled by a movable-rotatable semi-cylindrical battery. A number of results are reported in the literature regarding the dynamics of gliders steered by rudders or by movable masses. However, less results exist for gliders steered by rotatable masses. A rotatable mass has effect on both the mass and the inertial distribution, with the hydrodynamics different from other types of gliders. We first derive the dynamic model for the SIA glider following the approaches established by [8]-[10]. Based on the model, we solve for the parameters that characterize steady state spiraling motion in 3D. Our goal is to characterize and control the spiraling motion, which is shared by [9] and [13]. We follow a different approach aiming to find the exact solutions. This approach requires us to significantly simplify the nonlinear equations resulting from the glider model. The simplified equations lead naturally to a recursive algorithm that requires less effort to compute than solving the original equations directly using numerical methods.

\section{DYNAMICS OF THE SIA GLIDER}

The SIA glider is modeled as a rigid body with fixed wing and tail. The control mechanisms include a buoyancy pump and a movable-rotatable battery mass. The hull with wings and tail attached is approximately cylindrical, and the center of buoyancy (CB) is designed to coincide with the geometric center of the cylinder. We assign the body coordinate frame as $\boldsymbol{e}:\left(\boldsymbol{e}_{1}, \boldsymbol{e}_{2}, \boldsymbol{e}_{3}\right)$ with its origin $\boldsymbol{e}_{\boldsymbol{o}}$ fixed at CB. Let $\boldsymbol{e}_{1}$ lie along the longitudinal axis of the glider, and $\boldsymbol{e}_{2}$ lie in the wing plane pointing to the right when viewed along the direction of $\boldsymbol{e}_{1}, \boldsymbol{e}_{3}$ is $\boldsymbol{e}_{1} \times \boldsymbol{e}_{2}$, as shown in Fig.1. We denote the inertial frame as $\boldsymbol{E}:(\boldsymbol{i}, \boldsymbol{j}, \boldsymbol{k})$ with its origin at $\boldsymbol{E}_{\boldsymbol{o}}$. The vector $\mathbf{b}=\left[\begin{array}{lll}x & y & z\end{array}\right]^{T}$ is the position of the glider from the origin of the inertial frame to the origin of the body frame and the posture referred by the Euler angles of the glider in the inertial frame is $\boldsymbol{\theta}=\left[\begin{array}{lll}\phi & \theta & \psi\end{array}\right]^{T}$. The glider moves through the fluid with the translational velocity $\mathbf{V}=\left[\begin{array}{lll}V_{1} & V_{2} & V_{3}\end{array}\right]^{T}$ and the angular velocity $\boldsymbol{\Omega}=\left[\begin{array}{lll}p & q & r\end{array}\right]^{T}$, all expressed with respect to the body frame. The rotation matrix $\mathbf{R}_{E B}$ maps the velocity vector $\mathbf{V}$ from the body frame to $\mathbf{v}$ in the inertial frame as

$$
\begin{gathered}
\mathbf{v}=\dot{\mathbf{b}}=\mathbf{R}_{E B} \mathbf{V} \\
\dot{\boldsymbol{\theta}}=\left[\begin{array}{ccc}
1 & s \phi \tan \theta & c \phi \tan \theta \\
0 & c \phi & -s \phi \\
0 & s \phi \sec \theta & c \phi \sec \theta
\end{array}\right] \boldsymbol{\Omega}
\end{gathered}
$$

where we use the notation $c \cdot=\cos (\cdot)$ and $s \cdot=\sin (\cdot)$, and 


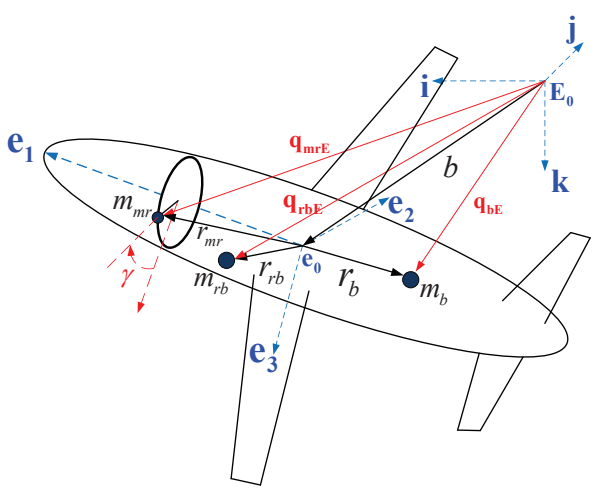

Fig. 1. Mechanical model of the SIA glider

$$
\mathbf{R}_{E B}=\left[\begin{array}{ccc}
c \theta c \psi & s \phi s \theta c \psi-c \phi s \psi & c \phi s \theta c \psi+s \phi s \psi \\
c \theta s \psi & c \phi c \psi+s \phi s \theta s \psi & -s \phi c \psi+c \phi s \theta s \psi \\
-s \theta & s \phi c \theta & c \phi c \theta
\end{array}\right]
$$

The stationary mass $m_{r b}$ includes the hull of the glider and other rigid body mass, the movable mass $m_{m r}$ represents the semi-cylindrical battery block, and the buoyancy mass is $m_{b}$. The total mass of the glider can then be expressed as follows

$$
m_{t}=m_{r b}+m_{m r}+m_{b}
$$

The mass of the displaced fluid is denoted as $m$. We define $\bar{m}=m_{t}-m$ so that the vehicle is negatively buoyant if $\bar{m}<0$ and is positively buoyant if $\bar{m}>0 . \mathbf{r}_{r b}, \mathbf{r}_{m r}$ and $\mathbf{r}_{b}$ are the positions of $m_{r b}, m_{m r}$ and $m_{b}$ in the body frame, respectively. The different masses and position vectors are illustrated in Fig.1. We define the operator $\hat{\cdot}$ as the skewsymmetric matrix constructed from a vector ', for threedimensional column vectors $\mathbf{x}$ and $\mathbf{y}$, we have $\hat{\mathbf{x}} \mathbf{y}=\mathbf{x} \times \mathbf{y}$.

\section{A. Equations of Motion}

We select $\mathbf{p}$ and $\boldsymbol{\pi}$ as the total translational momentum and the angular momentum of the glider/fluid system in the inertial frame. Let us assume that the translational velocity and angular velocity of the battery are $V_{m r}=0$ and $\omega_{m r}=$ 0 . As in [8], according to the Newton's laws ,we know that,

$$
\begin{aligned}
\dot{\mathbf{p}}= & \Sigma_{i=1}^{N_{1}} \mathbf{f}_{e x t i}+\left(m_{r b}+m_{m r}+m_{b}-m\right) g \mathbf{k} \\
\dot{\boldsymbol{\pi}}= & \mathbf{q}_{m r_{E}} \times m_{m r} g \mathbf{k}+\mathbf{q}_{b_{E}} \times m_{b} g \mathbf{k} \\
& +\mathbf{q}_{r b_{E}} \times m_{r b} g \mathbf{k}+\Sigma_{j=1}^{N_{2}} \boldsymbol{\tau}_{\text {ext } j}
\end{aligned}
$$

where $\mathbf{k}$ is a unit vector pointing to the direction of gravity, $\mathbf{f}_{\text {exti }}$ is the external force generated by the wing applied to the system, and $\boldsymbol{\tau}_{\text {extj }}$ is a pure external moment. $\mathbf{f}_{i n t k}$ is the force applied from the vehicle to the point mass $m_{m r}$. Vectors $\mathbf{q}_{m r E}, \mathbf{q}_{b E}$, and $\mathbf{q}_{r b E}$ denote the position of the centers of the battery mass, the buoyancy mass, and the rigid body mass in the inertial frame, respectively.

We analyze the generalized velocity and the generalized force of the glider in the body frame. First, we establish the relationship between the velocity and momentum of the gilder in the body frame as

$$
\eta=\mathbf{M} \nu
$$

where $\boldsymbol{\nu}=\left[\begin{array}{ll}\mathbf{V} & \boldsymbol{\Omega}\end{array}\right]$ are the translational velocity and angular velocity of the glider system in the body frame; $\boldsymbol{\eta}=\left[\begin{array}{ll}\mathbf{P}^{T} & \Pi^{T}\end{array}\right]^{T}$ are the generalized momentums of the glider in the body frame, $\mathbf{P}$ and $\boldsymbol{\Pi}$ are the translational momentum and the angular momentum relative to the gilder body frame. $\mathbf{M}$ is the generalized inertia matrix of the glider system. By differentiating (6) with respect to time, we get the relationship between the generalized force, velocity and acceleration as

$$
\dot{\eta}=\dot{\mathbf{M}} \nu+\mathbf{M} \dot{\nu}
$$

Next, we derive the total kinetic energy of the glider system to get $\mathbf{M}, \dot{\mathbf{M}}, \nu$, and $\dot{\nu}$.

As a stationary mass, the translational velocity and the angular velocity of the rigid body $m_{r b}$ in the body frame with respect to the origin $\mathbf{E}_{\boldsymbol{o}}$ can be expressed as

$$
\begin{aligned}
\mathbf{V}_{r b_{E}} & =\mathbf{V}+\boldsymbol{\Omega} \times \mathbf{r}_{r b} \\
& =\left[\begin{array}{ll}
\mathbf{I}_{3} & -\hat{\mathbf{r}}_{r b}
\end{array}\right]\left[\begin{array}{ll}
\mathbf{V}^{T} & \mathbf{\Omega}^{T}
\end{array}\right]^{T} \\
\boldsymbol{\Omega}_{r b_{E}} & =\boldsymbol{\Omega}
\end{aligned}
$$

The kinetic energy of the rigid body mass is expressed as $T_{r b}=\frac{1}{2} \boldsymbol{\nu}^{T} \mathbf{M}_{r b} \boldsymbol{\nu}$ where

$$
\mathbf{M}_{r b}=\left[\begin{array}{cc}
m_{r b} \mathbf{I}_{3} & -m_{r b} \hat{\mathbf{r}}_{r b} \\
m_{r b} \hat{\mathbf{r}}_{r b} & \mathbf{I}_{r b}-m_{r b} \hat{\mathbf{r}}_{r b} \hat{\mathbf{r}}_{r b}
\end{array}\right]
$$

where $\mathbf{I}_{r b}$ is the inertial matrix of the rigid body, and $\mathbf{I}_{3}$ is the identity matrix.

Similarly, the kinetic energy of the buoyancy mass $m_{b}$ is derived as follows $T_{b}=\frac{1}{2} \boldsymbol{\nu}^{T} \mathbf{M}_{b} \boldsymbol{\nu}$ where $\mathbf{I}_{b}$ is the inertial matrix of the buoyancy and

$$
\mathbf{M}_{b}=\left[\begin{array}{cc}
m_{b} \mathbf{I}_{3} & -m_{b} \hat{\mathbf{r}}_{b} \\
m_{b} \hat{\mathbf{r}}_{b} & \mathbf{I}_{b}-m_{b} \hat{\mathbf{r}}_{b} \hat{\mathbf{r}}_{b}
\end{array}\right]
$$

Accordingly, following [8], we express the kinetic energy generated by the added mass $\mathbf{M}_{f_{E}}$, the added inertia matrix $\mathbf{I}_{f_{E}}$ and cross term $\mathbf{C}_{f_{E}}$ due to the fluid effect as

$$
T_{f}=\frac{1}{2} \boldsymbol{\nu}^{T} \mathbf{M}_{f} \boldsymbol{\nu}
$$

where

$$
\mathbf{M}_{f}=\left[\begin{array}{cc}
\mathbf{M}_{f_{E}} & \mathbf{C}_{f_{E}} \\
\mathbf{C}_{f_{E}}^{T} & \mathbf{I}_{f_{E}}
\end{array}\right]
$$

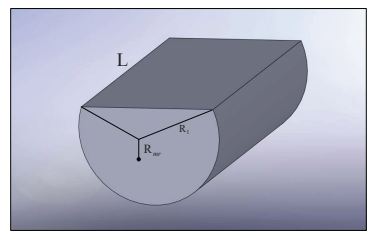

Fig. 2. Mechanical model of the battery

We model the battery mass $m_{m r}$ as a semi-cylinder block. The cross section of the glider's battery is shown in Fig.2 


$$
\left[\begin{array}{c}
\dot{\mathbf{V}} \\
\dot{\boldsymbol{\Omega}}
\end{array}\right]=\mathbf{M}^{-1}\left\{\left[\begin{array}{c}
\dot{\mathbf{P}}+\mathbf{P} \times \boldsymbol{\Omega} \bar{m} g\left(\mathbf{R}_{E B}^{T} \mathbf{k}\right)+\mathbf{F} \\
\dot{\boldsymbol{\Pi}}+\boldsymbol{\Pi} \times \boldsymbol{\Omega}+\mathbf{P} \times \mathbf{V}+\left(m_{m r} \mathbf{r}_{m r}+m_{r b} \mathbf{r}_{r b}+m_{b} \mathbf{r}_{b}\right) g \times\left(\mathbf{R}_{E B}^{T} \mathbf{k}\right)+\mathbf{T}
\end{array}\right]\right\}
$$

with radius $R_{1}$, length $\mathrm{L}$, and the eccentric distance $R_{m r}$. In order to tune the roll and the pitch of the glider, the battery is controlled to move and rotate along the longitudinal axis $\boldsymbol{e}_{1}$. When the battery mass rotates around $\boldsymbol{e}_{1}$ with an angle $\gamma$, the inertia matrix of the semi-cylinder block is a function of $\gamma$ as

$$
\mathbf{I}_{m r}(\gamma)=\mathbf{R}_{e 1}^{T}(\gamma) \mathbf{I}_{m r}^{0} \mathbf{R}_{e 1}(\gamma)
$$

where

$$
\mathbf{R}_{e 1}=\left[\begin{array}{ccc}
1 & 0 & 0 \\
0 & \cos \gamma & -\sin \gamma \\
0 & \sin \gamma & \cos \gamma
\end{array}\right]
$$

and $\mathbf{I}_{m r}^{0}$ represents the principal inertia matrix of the semicylinder computed at the stationary state with $\gamma=0$. Since the semi-cylinder mass $m_{m r}$ is constrained to move and rotate along the longitudinal axis $\boldsymbol{e}_{1}$, we denote vector $\mathbf{r}_{m r x}$ as the position in axis $\boldsymbol{e}_{1}$. Hence, the position $\mathbf{r}_{m r}$ and the angle velocity $\boldsymbol{\Omega}_{m r}$ in the body frame are expressed as

$$
\begin{array}{r}
\mathbf{r}_{m r}=r_{m r x} \boldsymbol{e}_{1}+R_{m r}\left(\cos (\gamma+\pi / 2) \boldsymbol{e}_{2}+\sin (\gamma+\pi / 2) \boldsymbol{e}_{3}\right) \\
\boldsymbol{\Omega}_{m r}=\dot{\gamma} \boldsymbol{e}_{1}
\end{array}
$$

We differentiate $\mathbf{r}_{m r}$ to get the velocity $\mathbf{V}_{m r}$ of the semicylinder mass $m_{m r}$ in the body frame as follows

$$
\begin{aligned}
\dot{\mathbf{r}}_{m r} & =\dot{r}_{m r x} \boldsymbol{e}_{1}+R_{m r} \dot{\gamma}\left(-\cos \gamma \boldsymbol{e}_{2}-\sin \gamma \boldsymbol{e}_{3}\right) \\
& =\mathbf{V}_{m r x}-\hat{\mathbf{r}}_{m r} \boldsymbol{\Omega}_{m r}
\end{aligned}
$$

In order to get the kinetic energy of the battery mass, we derive the absolute velocity of the battery mass with respect to the origin of the inertial frame. The velocity of the the battery block is defined as $\mathbf{V}_{m r_{E}}$ in body frame with respect to the origin $\mathbf{E}_{\boldsymbol{o}}$. The vector $\mathbf{L}_{m r}$ denotes the position of the point mass $m_{r b}$ in the inertial frame relative to the origin $\mathbf{e}_{\boldsymbol{o}}$.

$$
\mathbf{r}_{m r}=\mathbf{R}_{E B}^{T} \mathbf{L}_{m r}=\mathbf{R}_{E B}^{T}\left(\mathbf{q}_{m r_{E}}-\mathbf{b}\right)
$$

Differentiating (15), we express the relationship between the velocity $\mathbf{V}_{m r}$ and the absolute velocity $\mathbf{V}_{m r_{E}}$ as

$$
\begin{aligned}
\mathbf{V}_{m r} & =\dot{\mathbf{R}}_{E B}^{T} \mathbf{L}_{m r}+\mathbf{R}_{E B}^{T}\left(\dot{\mathbf{q}}_{m r_{E}}-\dot{\mathbf{b}}\right) \\
& =\hat{\mathbf{r}}_{m r} \boldsymbol{\Omega}+\mathbf{V}_{m r_{E}}-\mathbf{V}
\end{aligned}
$$

Combing (16) and (14) to cancel the term $\dot{r}_{m r}$, we get the velocity $\mathbf{V}_{m r E}$ and the angular velocity $\boldsymbol{\Omega}_{m r E}$ of the battery mass $m_{m r}$ as

$$
\begin{aligned}
\mathbf{V}_{m r_{E}} & =\mathbf{V}-\mathbf{V}_{m r} \boldsymbol{\Omega}+\dot{\mathbf{r}}_{m r x}-\hat{\mathbf{r}}_{m r} \boldsymbol{\Omega}_{m r} \\
\boldsymbol{\Omega}_{m r_{E}} & =\boldsymbol{\Omega}_{m r}+\boldsymbol{\Omega}
\end{aligned}
$$

When the mass rotates slowly, we can neglect $\mathbf{V}_{m r}$ and $\boldsymbol{\Omega}_{m r}$, The translational velocity and the angular velocity can be expressed as

$$
\begin{aligned}
\mathbf{V}_{m r_{E}} & =\mathbf{V}-\hat{\mathbf{r}}_{m r} \boldsymbol{\Omega} \\
\boldsymbol{\Omega}_{m r_{E}} & =\boldsymbol{\Omega}
\end{aligned}
$$

So the kinetic energy of the battery mass is expressed as:

$$
\begin{aligned}
T_{m r} & =\frac{1}{2} m_{m r}\left\|\mathbf{V}_{m r_{E}}\right\|^{2}+\frac{1}{2} \boldsymbol{\Omega}_{m r_{E}}^{T} \cdot \mathbf{I}_{m r}(\gamma) \boldsymbol{\Omega}_{m r_{E}} \\
& =\frac{1}{2} \boldsymbol{\nu}^{T} \mathbf{M}_{m r} \boldsymbol{\nu}
\end{aligned}
$$

Then we get the total energy of the SIA glider system as:

$$
\begin{aligned}
T & =T_{m r}+T_{r b}+T_{b}+T_{f} \\
& =\frac{1}{2} \boldsymbol{\nu}^{T} \mathbf{M} \boldsymbol{\nu}
\end{aligned}
$$

The generalized inertia matrix is

$$
\mathbf{M}=\left[\begin{array}{ll}
\mathbf{M}_{t} & \mathbf{C}_{t} \\
\mathbf{C}_{t}^{T} & \mathbf{I}_{t}
\end{array}\right]
$$

where $\mathbf{M}_{t}=\left(m_{m r}+m_{r b}+m_{b}\right) \mathbf{I}_{3}+\mathbf{M}_{f E}, \mathbf{C}_{t}=\mathbf{C}_{f}-$ $m_{r b} \hat{\mathbf{r}}_{r b}-m_{b} \hat{\mathbf{r}}_{b}-m_{m r} \hat{\mathbf{r}}_{m r}$, and $\mathbf{I}_{t}=\mathbf{I}_{r b}+\mathbf{I}_{b}+\mathbf{I}_{m r}(\gamma)+$ $\mathbf{I}_{f_{E}}-m_{m r} \hat{\mathbf{r}}_{m r} \hat{\mathbf{r}}_{m r}-m_{r b} \hat{\mathbf{r}}_{r b} \hat{\mathbf{r}}_{r b}-m_{b} \hat{\mathbf{r}}_{b} \hat{\mathbf{r}}_{b}$.

And then, based on (5), we map the generalized momentum $\left[\begin{array}{ll}\mathbf{p}^{T} & \boldsymbol{\pi}^{T}\end{array}\right]^{T}$ from the inertial frame to the generalized momentum $\boldsymbol{\eta}$ in the body frame as

$$
\begin{aligned}
\mathbf{p} & =\mathbf{R}_{E B} \mathbf{P} \\
\boldsymbol{\pi} & =\mathbf{R}_{E B} \boldsymbol{\Pi}+\mathbf{b} \times \mathbf{p}
\end{aligned}
$$

Differentiating (20) and using the kinematic matrix (1) and (2), we get the relationship among $\boldsymbol{\eta}, \boldsymbol{\nu}, \dot{\boldsymbol{\nu}}$, and $\dot{\boldsymbol{\eta}}$ as

$$
\begin{aligned}
\dot{\mathbf{p}} & =\mathbf{R}_{E B}(\dot{\mathbf{P}}+\hat{\mathbf{\Omega}} \mathbf{P}) \\
\dot{\boldsymbol{\pi}} & =\mathbf{R}_{E B}(\dot{\boldsymbol{\Pi}}+\hat{\mathbf{\Omega}} \boldsymbol{\Pi})+\mathbf{R}_{E B} \mathbf{V} \times \mathbf{p}+\mathbf{b} \times \dot{\mathbf{p}}
\end{aligned}
$$

Substituting (21) into (5), we obtain

$$
\begin{aligned}
\dot{\mathbf{P}}= & \mathbf{P} \times \boldsymbol{\Omega}+\bar{m} g\left(\mathbf{R}_{E B}^{T} \mathbf{k}\right)+\mathbf{R}_{E B}^{T} \Sigma_{i=1}^{N_{1}} \mathbf{f}_{e x t i} \\
\dot{\Pi}= & \boldsymbol{\Pi} \times \boldsymbol{\Omega}+\mathbf{P} \times \mathbf{V}+\mathbf{R}_{E B}^{T} \Sigma_{j=1}^{N_{2}} \boldsymbol{\tau}_{e x t j} \\
& +\left(m_{r b} \mathbf{r}_{r b}+m_{m r} \mathbf{r}_{m r}+m_{b} \mathbf{r}_{b}\right) g \times\left(\mathbf{R}_{E B}^{T} \mathbf{k}\right)(22)
\end{aligned}
$$

Then we substitute (22) into (7), and at last we get the total dynamic model as in (26), where $\mathbf{F}=\mathbf{R}_{E B}^{T}{ }^{\Sigma_{i=1}^{N}} \mathbf{f}_{\text {etxti }}$, and $\mathbf{T}=\mathbf{R}_{E B}^{T} \Sigma_{j=1}^{N_{2}} \boldsymbol{\tau}_{e x t j}$. 
$0=V \omega_{3}\left(m_{t 2} \sin \beta \cos \phi \cos \theta-m_{t 3} \sin \alpha \cos \beta \sin \phi \cos \theta\right)$

$+m_{m r} \omega_{3}^{2}\left(r_{m r x} \cos ^{2} \theta+R_{m r} \frac{\sin 2 \theta}{2} \cos (\phi+\gamma)\right)-\bar{m} g \sin \theta-D \cos \alpha \cos \beta-S F \cos \alpha \sin \beta+L \sin \alpha$

$0=V \omega_{3}\left(-m_{t 3} \sin \alpha \cos \beta \sin \theta-m_{t 1} \cos \alpha \cos \beta \cos \phi \cos \theta\right)+\bar{m} g \sin \phi \cos \theta-D \sin \beta+S F \cos \beta$

$+m_{m r} \omega_{3}^{2}\left(\frac{r_{m r x} \sin 2 \theta \sin \phi}{2}-\frac{R_{m r} \sin 2 \phi \cos ^{2} \theta \cos \gamma}{2}-R_{m r} \sin \gamma\left(\cos ^{2} \phi \cos ^{2} \theta+\sin ^{2} \theta\right)\right)$

$0=\left(I_{m r y}-I_{m r z}-m_{m r} R_{m r}^{2}\right) \omega_{3}^{2} \frac{\sin 2 \gamma \sin \phi \sin 2 \theta}{4}+\left(M_{f 3}-M_{f 1}\right) V^{2} \cos ^{2} \beta \frac{\sin 2 \alpha}{2}$

$-m_{m r} V \omega_{3} r_{m r x}(\cos \alpha \cos \beta \sin \phi \cos \theta+\sin \theta \sin \beta)-m_{m r} R_{m r} r_{m r x} \omega_{3}^{2} \frac{\sin 2 \phi \cos ^{2} \theta \sin \gamma}{2}$

$+\left(I_{r b x}-I_{r b z}+I_{m r x}+I_{f 1}-I_{f 3}-I_{m r y} \sin ^{2} \gamma+\left(m_{m r} R_{m r}^{2}-I_{m r z}\right) \cos ^{2} \gamma-m_{m r} r_{m r x}^{2}\right) \omega_{3}^{2} \cos \phi \frac{\sin 2 \theta}{2}$

$-m_{m r} g r_{m r x} \cos \phi \cos \theta+M_{D L 1} \sin \beta+M_{D L 2} \cos \beta+m_{m r} V R_{m r} \omega_{3} \cos \gamma \cos \theta(\sin \beta \cos \phi-\sin \phi \sin \alpha \cos \beta)$

$\cos \gamma\left(-m_{m r} g R_{m r} \sin \theta+m_{m r} R_{m r} r_{m r x} \omega_{3}^{2}\left(\cos ^{2} \phi \cos ^{2} \theta-\sin ^{2} \theta\right)\right)$

$$
\begin{aligned}
& f_{\gamma 1}=m_{m r} V R_{m r} \omega_{3} \cos \theta(\sin \phi \sin \alpha \cos \beta-\sin \beta \cos \phi)-m_{m r} R_{m r} r_{m r x} \omega_{3}^{2}\left(\cos ^{2} \phi \cos ^{2} \theta-\sin ^{2} \theta\right)+m_{m r} g R_{m r} \sin \theta \\
& f_{\gamma 2}=m_{m r} R_{m r} r_{m r x} \omega_{3}^{2} \cos \phi \cos ^{2} \theta \sin \phi \\
& f_{\gamma}=\left(I_{m r y}-I_{m r z}-m_{m r} R_{m r}^{2}\right) \omega_{3}^{2} \frac{\sin 2 \gamma \sin \phi \sin 2 \theta}{4}-m_{m r} r_{m r x} \omega_{3} V(\cos \alpha \cos \beta \sin \phi \cos \theta+\sin \theta \sin \beta) \\
& +\left(I_{r b x}-I_{r b z}+I_{m r x}+I_{f 1}-I_{f 3}-I_{m r y} \sin ^{2} \gamma+\left(m_{m r} R_{m r}^{2}-I_{m r z}\right) \cos ^{2} \gamma-m_{m r} r_{m r x}^{2}\right) \omega_{3}^{2} \cos \phi \frac{\sin 2 \theta}{2} \\
& -m_{m r} g r_{m r x} \cos \phi \cos \theta+\sin \beta M_{D L 1}+\cos \beta M_{D L 2}+\left(M_{f 3}-M_{f 1}\right) V^{2} \cos ^{2} \beta \frac{\sin 2 \alpha}{2}
\end{aligned}
$$

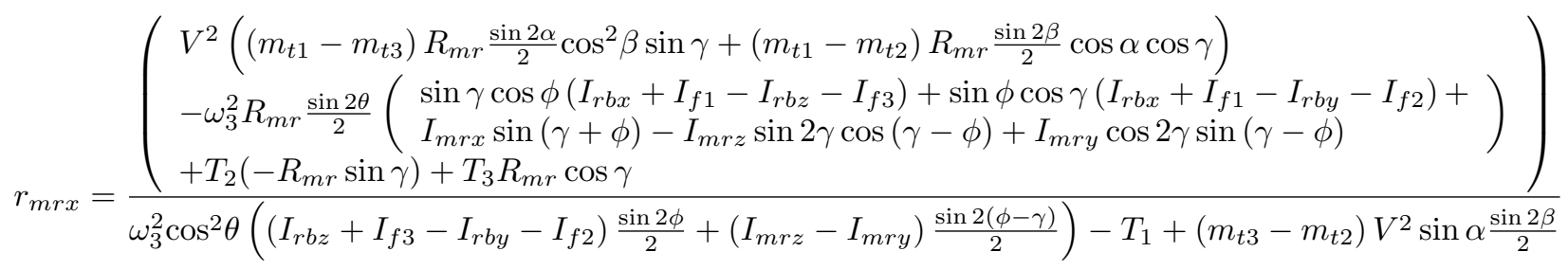

\section{B. Hydrodynamic forces on the glider}

In this section, the hydrodynamic forces $\mathbf{F}$ and $\mathbf{T}$ are first expressed as functions of the hydrodynamic angles $\alpha$ and $\beta$, which is defined by

$$
\alpha=\tan ^{-1}\left(\frac{V_{3}}{V_{1}}\right), \beta=\sin ^{-1}\left(\frac{V_{2}}{V}\right), V=\|\mathbf{V}\|
$$

According to [9], the hydrodynamic force $\mathbf{F}_{h y}$ and moment $\mathbf{M}_{D L}$ are simplified as

$$
\begin{aligned}
\mathbf{F}_{h y}= & {\left[\begin{array}{c}
-D \\
S F \\
-L
\end{array}\right]=\left[\begin{array}{c}
-\left(K_{D 0}+K_{D} \alpha^{2}\right) V^{2} \\
K_{\beta} \beta V^{2} \\
-\left(K_{L 0}+K_{L} \alpha\right) V^{2}
\end{array}\right] } \\
\mathbf{M}_{D L}= & {\left[\begin{array}{c}
\left(K_{M R} \beta+K_{p} p\right) V^{2} \\
\left(K_{M 0}+K_{M} \alpha+K_{q} q\right) V^{2} \\
\left(K_{M Y} \beta+K_{r} r\right) V^{2}
\end{array}\right] }
\end{aligned}
$$

and then $\mathbf{F}$ and $\mathbf{T}$ are expressed as

$$
\mathbf{F}=\mathbf{R}_{B C} \mathbf{F}_{h y}, \mathbf{T}=\mathbf{R}_{B C} \mathbf{M}_{D L}
$$

where

$$
\mathbf{R}_{B C}=\left[\begin{array}{ccc}
c \alpha c \beta & -c \alpha s \beta & -s \alpha \\
s \beta & c \beta & 0 \\
s \alpha c \beta & -s \alpha s \beta & c \alpha
\end{array}\right]
$$

The Hydrodynamic coefficients depend on the glider speed, geometry, and the Reynolds number. CFD, Fluent and other software or towing trials can be used to determine the hydrodynamic coefficients. The added mass and inertial terms, which are included in the added inertial matrix $\mathbf{M}_{f_{E}}$, $\mathbf{I}_{f_{E}}$ and the added hydrodynamic coriolis and centripetal matrix $\mathbf{C}_{f_{E}}$, are given based on analytical method of the strip theory. When the coefficients are known, since $\alpha$ and $\beta$ are completely determined by $\mathrm{V}$, the hydrodynamic forces and torques can be viewed as functions of $V$, i.e. $\mathbf{F}(V), \mathbf{T}(V)$.

\section{GLIDER MOTION IN THREE DIMENSION}

The steady state glider motion includes the 3D spiraling motion. Assuming that the position of the battery is fixed at $\mathbf{r}_{m r}$ and with a constant buoyancy mass, which imply that the control $\dot{m}_{b}=0$, the glider equilibria equations in three dimension are 


$$
\begin{gathered}
0=\mathbf{P} \times \boldsymbol{\Omega}+\bar{m} g\left(\mathbf{R}_{E B}^{T} \mathbf{k}\right)+\mathbf{F} \\
0=\boldsymbol{\Pi} \times \mathbf{\Omega}+\mathbf{P} \times \mathbf{V}+m_{m r} g \mathbf{r}_{m r} \times\left(\mathbf{R}_{E B}^{T} \mathbf{k}\right)+\mathbf{T}
\end{gathered}
$$

After expanding the force equation along $\boldsymbol{e}_{1}$ and $\boldsymbol{e}_{2}$ in (25), and moment equation around $\boldsymbol{e}_{1}$ in (26), we get (27), (28) and (29). We consider the case when the buoyancy mass and the rigid body mass produce the combined effect such that the center of buoyancy of the glider can be studied by letting $r_{r b}=0$ and $r_{b}=0$. Then $\mathbf{P}$ and $\boldsymbol{\Pi}$ are simplified as

$$
\begin{aligned}
& \mathbf{P}=\mathbf{M}_{\mathbf{t}} \mathbf{V}-m_{m r} \hat{\mathbf{r}}_{\mathbf{m r}} \boldsymbol{\Omega} \\
& \boldsymbol{\Pi}=m_{m r} \hat{\mathbf{r}}_{\mathbf{m r}} \mathbf{V}+\mathbf{I}_{\mathbf{t}} \boldsymbol{\Omega}
\end{aligned}
$$

The steady state vertical motion has been throughly investigated in [8]. In this section, we analyze the steady spiraling motion where the yaw of the glider changes at a constant rate while the pitch and roll angles are constant. This implies that $\mathbf{R}_{E B}^{T} \mathbf{k}$ are constant. By taking the time derivative of $\mathbf{R}_{E B}^{T} \mathbf{k}$, we get the result that

$$
\boldsymbol{\Omega} \times\left(\mathbf{R}_{E B}^{T} \mathbf{k}\right)=0
$$

From (32), we know that the underwater glider moves with constant speed along a circular helix which is aligned with the direction of gravity, hence the angular velocity is $\boldsymbol{\Omega}=\mathbf{R}_{E B}^{T} \mathbf{k} \omega_{3}$. The spiraling motion can be projected into rotational motion around $\mathbf{k}$ and an ascending motion along $\mathbf{k}$, we analyze the rotational motion and then ascending motion by projecting the total velocity $V$ into the turning direction and the vertical direction as

$$
\begin{aligned}
& V \cos (\theta-\alpha)=\omega_{3} R \\
& V \sin (\theta-\alpha)=V_{\text {vertical }}
\end{aligned}
$$

The steady spiraling motion can be determined by ten parameters: $V, \alpha, \beta$ which describe the velocity of the glider, $\omega_{3}, \phi, \theta$ which describe the glider angular velocity, the controllable buoyancy $m_{b}$, the battery position $r_{m r x}$ and $\gamma$, and the turning radius $R$. By combining (25), (26) and (33), we obtain seven equations of the 3D spiral motion. Since the equations may not be independent, we need to specify at least three parameter out of ten to solve for the rest of the seven parameters.

We consider the situation where $V, \alpha, \beta$ are known, from which we know the expression of $\mathbf{T}, \mathbf{F}$, and $\mathbf{R}_{B C}$, then we solve for the other seven parameters. In order to make (25) and (26) simpler to solve, we take the inner products with respect to $\Omega$ on both sides of (25), (26) and take the inner products on both sides of (26) with respect to $\mathbf{r}_{m r}$, then we get

$$
\begin{aligned}
0 & =\frac{\bar{m} g}{\omega_{3}} \mathbf{\Omega} \bullet \mathbf{\Omega}+\mathbf{F} \bullet \mathbf{\Omega} \\
0 & =(\mathbf{P} \times \mathbf{V}+\mathbf{T}) \bullet \mathbf{\Omega} \\
0 & =\left(\mathbf{I}_{\mathbf{t}} \mathbf{\Omega} \times \mathbf{\Omega}\right) \bullet \mathbf{r}_{\mathbf{m r}}+\left(\mathbf{M}_{\mathbf{t}} \mathbf{V} \times \mathbf{V}\right) \bullet \mathbf{r}_{\mathbf{m r}}+\mathbf{T} \bullet \mathbf{r}_{\mathbf{m r}}
\end{aligned}
$$

From (35), we can solve for the buoyancy mass $\bar{m}$ as

$$
\bar{m}=\frac{-\mathbf{F} \bullet\left(\mathbf{R}_{E B}^{T} \mathbf{k}\right)}{g}
$$

Using (36) and (25), we can get the glider rotate velocity $\omega_{3}$ as

$$
\omega_{3}=-\frac{\left(\mathbf{F}+\bar{m} g \mathbf{R}_{E B}^{T} \mathbf{k}\right) \bullet \mathbf{V}}{\mathbf{T} \bullet\left(\mathbf{R}_{E B}^{T} \mathbf{k}\right)}
$$

Note that $\mathbf{T}$ is also a function of $\omega_{3}$. Hence (39) is a nonlinear equation for $\omega_{3}$, which can be solved analytically if $\theta, \phi$ are known. Therefore, supposing solutions of $\theta, \phi$ are found, we can find $\bar{m}$ and $\omega_{3}$ from (38) and (39). After that, we can solve for turning radius from (33) as

$$
R=\frac{V \cos (\theta-\alpha)}{\omega_{3}}
$$

With the assumption that we know $V, \alpha$, and $\beta$, we can solve for $\bar{m}, \omega_{3}$ and $R$ as functions of $\theta$ and $\phi$. This process has reduced the unknown parameters from seven to four. We still need solve for $\theta, \phi, \gamma$, and $r_{m r x}$. When $\omega_{3}$ is small, (27) and (28) can be used to solve for $\theta$ and $\phi$. Then (29) and (37) can be used to solve for $\gamma$ and $r_{m r x}$. When $\omega_{3}$ is not small, (27), (28), (29),(37), (38), and (39) have to be solved altogether. We define a recursive vector as $\Delta=\left[\theta, \phi, \gamma, r_{m r x}, R, \bar{m}, \omega_{3}\right]$, we next establish the recursive equations to solve $\Delta$ as

$$
\Delta^{k}=F\left(\Delta^{k-1}\right)
$$

From (27), we solve for $\theta$ as

$$
\theta=\arcsin \frac{f_{\theta}}{\sqrt{f_{\theta 1}^{2}+f_{\theta 2}^{2}}}-\lambda_{\theta}
$$

where

$$
\begin{aligned}
f_{\theta 1} & =V \omega_{3}\left(m_{t 2} \sin \beta \cos \phi-m_{t 3} \sin \alpha \cos \beta \sin \phi\right) \\
f_{\theta 2} & =-\bar{m} g \\
f_{\theta} & =D \cos \alpha \cos \beta+S F \cos \alpha \sin \beta-L \sin \alpha \\
& -m_{m r} \omega_{3}^{2}\left(r_{m r x} \cos ^{2} \theta+R_{m r} \frac{\sin 2 \theta}{2} \cos (\phi+\gamma)\right) \\
\sin \lambda_{\theta} & =\frac{f_{\theta 1}}{\sqrt{f_{\theta 1}^{2}+f_{\theta 2}^{2}}}, \cos \lambda_{\theta}=\frac{f_{\theta 2}}{\sqrt{f_{\theta 1}^{2}+f_{\theta 2}^{2}}}
\end{aligned}
$$

Similarly, from (28), we express the roll angle $\phi$ as

$$
\phi=\arcsin \frac{f_{\phi}}{\sqrt{f_{\phi 1}^{2}+f_{\phi 2}^{2}}}-\lambda_{\phi}
$$

where

$$
\begin{aligned}
f_{\phi 1}= & -\bar{m} g \cos \theta-m_{m r} \omega_{3}^{2} r_{m r x} \frac{\sin 2 \theta}{2} \\
f_{\phi 2}= & V \omega_{3} m_{t 1} \cos \alpha \cos \beta \cos \theta \\
f_{\phi}= & S F \cos \beta-D \sin \beta-V \omega_{3} m_{t 3} \sin \alpha \cos \beta \sin \theta \\
& +m_{m r} \omega_{3}^{2}\left(-\frac{R_{m r} \sin 2 \phi \cos ^{2} \theta \cos \gamma}{2}\right. \\
& \left.-R_{m r} \sin \gamma\left(\cos ^{2} \phi \cos ^{2} \theta+\sin ^{2} \theta\right)\right) \\
\sin \lambda_{\phi}= & \frac{f_{\phi 2}}{\sqrt{f_{\phi 1}^{2}+f_{\phi 2}^{2}}}, \cos \lambda_{\phi}=\frac{f_{\phi 1}}{\sqrt{f_{\phi 1}^{2}+f_{\phi 2}^{2}}}
\end{aligned}
$$


When $\omega_{3}^{2}$ is quite small, the terms that contains $r_{m r}$ and $\gamma$ disappear from (42) and (43). Hence after we replace $\bar{m}$ by (38), the equations (39), (42), and (43) can be solved recursively for $\theta$ and $\phi$.

We next derive the recursive equations for $r_{m r x}$ and $\gamma$. From (29), we express the battery angle $\gamma$ as

$$
\gamma=\arcsin \frac{f_{\gamma}}{\sqrt{f_{\gamma_{1}}^{2}+f_{\gamma_{1}}^{2}}}-\lambda_{\gamma}
$$

Where $\sin \lambda_{\gamma}=\frac{f_{\gamma_{1}}}{\sqrt{f_{\gamma_{1}}^{2}+f_{\gamma_{2}}^{2}}}, \cos \lambda_{\gamma}=\frac{f_{\gamma_{2}}}{\sqrt{f_{\gamma_{1}}^{2}+f_{\gamma_{2}}^{2}}}$, and $f_{\gamma_{1}}, f_{\gamma_{2}}, f_{\gamma}$ are defined in (45). From (37), we get the battery translational postion $r_{m r x}$ as (46). Therefore, we have shown that the solution of the steady spiraling motion can be derived using a recursive algorithm developed from (38), (39), (40), (42), (43), (44), and (46).

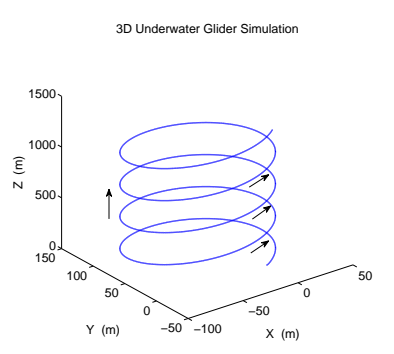

(a) A spiraling motion

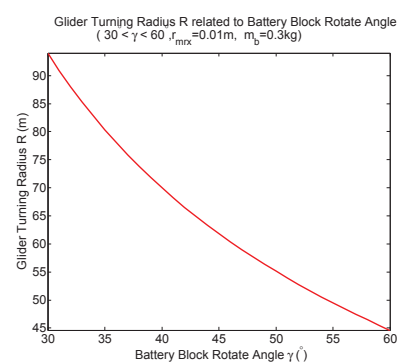

(b) Relationship between $R$ and $\gamma$
Fig. 3. The simulation results for 3D spiraling motion.

\section{SIMULATION}

In this section, we give the simulation results demonstrated by a glider simulation program developed by SIA where hydrodynamic effects are considered. We then compare the results with the solutions obtained by the recursive algorithm for the spiraling motion. The rigid body mass and the battery block mass are $m_{r b}=54.28 \mathrm{~kg}$ and $m_{m r}=11 \mathrm{~kg}$, and the volume displacement mass of the SIA glider is $m=65.28 \mathrm{~kg}$, the moving range and the rotation range of the battery block mass are $-0.05 m<r_{m r x}<0.05 m,-90^{\circ}<\gamma<90^{\circ}$, and the range of buoyancy adjustment is $-0.8 \mathrm{~kg}<m_{b}<0.8 \mathrm{~kg}$. The simulation result are shown in Fig. 3. The relationship between the battery angle and the glider turning radius is shown in Fig. 3(b). When the block mass has a rotation angle $\gamma=45^{\circ}$, we get a spiraling motion equilibrium in Fig.3(a) with $V=0.503 \mathrm{~m} / \mathrm{s}, \alpha=1.111^{\circ}, \beta=-1.839^{\circ}$, $\omega_{3}=0.0064 \mathrm{rad} / \mathrm{s}, \phi=-21.314^{\circ}, \theta=-37.59^{\circ}$, and the radius of the spiraling motion is $R \approx 61.83$. With the assumption that $V, \alpha, \beta$ are known, we initiate the recursive algorithm from the vertical gliding motion parameters $m_{b}=0.001 \mathrm{~kg}, r_{m r x}=0.001 \mathrm{~m}, \gamma=0.001^{\circ}, \omega_{3}=$ $0.001 \mathrm{rad} / \mathrm{s}, \phi=-0.001^{\circ}, \theta=-0.001^{\circ}$. Then the algorithm converges to the solutions as $m_{b}=0.3 \mathrm{~kg}, r_{m r x}=$ $0.011 \mathrm{~m}, \gamma=39.42^{\circ}, \omega_{3}=0.0067 \mathrm{rad} / \mathrm{s}, \phi=-21.22^{\circ}, \theta=$ $-37.59^{\circ}, R=58.66 \mathrm{~m}$. The errors between the recursive solutions and the simulation results are less than $5 \%$ for each parameter.

\section{CONCLUSIONS AND FUTURE WORKS}

We analyze the steady state spiraling motion of the SIA glider by solving for the parameters that completely characterize the spiraling motion. A recursive algorithm is devised after the motion equations are significantly simplified to find the exact solutions. Future works include analysis of the convergence conditions for the recursive algorithm, and comparisons between experimental and analytical results.

\section{REFERENCES}

[1] D.C.Webb, P.J.Simonetti, and C.P.Jones, "SLOCUM, an underwater glider propelled by environmental energy," IEEE Journal of Oceanic Engineering , vol. 26, 2001, pp. 447-452.

[2] J.Sherman, R.E.Davis, W.B.Owens, and J. Valdes, "The autonomous underwater glider "Spray"," IEEE Journal of Oceanic Engineering, vol. 26, 2001, pp. 437-446.

[3] C.C.Eriksen, T.J.Osse, R.D.Light, T.Wen, T.W.Lehman, P.L.Sabin, J.W.Ballard, and A.M.Chiodi, "Seaglider: A long range autonomous underwater vehicle for oceanographic research," IEEE Journal of Oceanic Engineering, vol. 26, 2001, pp. 424-436.

[4] T.J.Osse and C.C.Eriksen, "The Deepglider: A Full Ocean Depth Glider for Oceanographic Research," in IEEE Conference Oceans'2007, Sept. 2007, pp.1-12.

[5] R.N.Smith, Y.Chao, P.P.Li, D.A.Caron, B.H.Jones and G.S.Sukhatme, "Planning and implementing trajectories for autonomous underwater vehicles to track evolving ocean processes based on predictions from a regional ocean model," International Journal of Robotics Research , 2010, pp.1-23.

[6] D.L.Rudnick, R.E.Davis, and D.M.Fratantoni, "Underwater Gliders for Ocean Research,” Marine Technology Society Journal, vol.38, 2004, pp.73-84.

[7] S.A.Jenkins, D.E.Humphreys, J.Sherman, J.Osse, C.Jones, N.Leonard, J.Graver, and R. Bachmayer, "Underwater Glider System Study," UC San Diego: Scripps Institution of Oceanography, 2003.

[8] N.E.Leonard and J.Graver, "Model-Based Feedback Control of Autonomous Underwater Gliders,' IEEE Journal of Oceanic Engineering, vol. 26, 2001, pp.633-645.

[9] P.Bhatta and N.E.Leonard, "Nonlinear Gliding Stability and Control for Vehicles with Hydrodynamic Forcing," Automatica, vol. 44, 2008, pp. $1240-1250$.

[10] J.Graver and N.E.Leonard, "Underwater Glider Dynamics and Control," in 12th International Symposium on Unmanned Untethered Submersible Technology, 2001.

[11] A.Bender, D.M.Steinberg, A.L.Friedman, and S.B.Williams, "Analysis of an Autonomous Underwater Glider," in Australasian Conference on Robotics and Automation, 2008, pp.1-10.

[12] N.Mahmoudian, J.Geisbert, and C.Woolsey, "Approximate Analytical Turning Conditions for Underwater Gliders: Implications for Motion Control and Path Planning," IEEE Journal of Oceanic Engineering , vol. 35, 2010, pp.131-143.

[13] N.Mahmoudian and C.Woolsey, "Underwater Glider Motion Control," in Proceedings of the 47th IEEE Conference on Decision and Control , Dec, 2008, pp.552 - 557.

[14] J.S.Geisbert, "Hydrodynamic Modeling for Autonomous Underwater Vehicles Using Computational and Semi-Empirical Methods," Master's thesis, Virginia Polytechnic Institute and State University, 2007.

[15] K.A.Morgansen, B.I.Triplett, and D.J.Klein, "Geometric Methods for Modeling and Control of Free-Swimming Fin-Actuated Underwater Vehicles," IEEE Transactions on Robotics, vol.23, 2007, pp.11841199.

[16] P.Jagadeesh, K.Murali and V.G.Idichandy, "Experimental investigation of hydrodynamic force coefficients over AUV hull form," Ocean Engineering , vol.36, 2009, pp.113-118.

[17] M.Arima, N.Ichihashi, and Y.Miwa, "Modelling and motion simulation of an underwater glider with independently controllable main wings," in IEEE Conference Oceans'2009, May 2009, pp.1-6.

[18] Jiancheng Yu, Zhiqiang Hu, Haitao Gu, Fumin Zhang, "Modelling and motion simulation of an underwater glider with independently controllable main wings," in Proceedings of the Twentieth (2010) International Offshore and Polar Engineering Conference, June 2010, pp.295-301. 